\title{
Apparent Diffusion Coefficient and Time Intensity Curve Values from Dynamic Contrast Enhancement (DCE) MRI in Osteosarcoma Histopathological Subtypes
}

\author{
Bagus Novariyanto ${ }^{\mathrm{a}}$, Rosy Setiawati ${ }^{\mathrm{a}}$, Paulus Rahardjo ${ }^{\mathrm{a}}$, Sjahjenny Mustokoweni $^{\mathrm{b}}$, Giuseppe Guglielmi ${ }^{\mathrm{c}}$ \\ *Corresponding author, rosy-s@fk.unair.ac.id \\ ${ }^{a}$ Department of Radiology, Faculty of Medicine, Universitas Airlangga, Surabaya, Indonesia \\ ${ }^{b}$ Department of Anatomical Pathology, Faculty of Medicine, Universitas Airlangga, Surabaya, Indonesia \\ 'Department of Radiology, School of Medicine, Foggia University, Foggia, Italy
}

\begin{abstract}
Background: According to WHO criteria osteosarcoma consists of various histopathological subtypes. Magnetic resonance imaging (MRI) is an important imaging modality for preoperative and post-treatment evaluations of osteosarcoma. Dynamic Contrast Enhancement Magnetic Resonance Imaging (DCE-MRI) study is used to determine Apparent Diffusion Coefficient (ADC) value and Time Intensity Curve (TIC). This study aims to describe Apparent Diffusion Coefficient value and Time Intensity Curve in osteosarcoma of histopathological subtypes.

Methods: This study was a retrospective with observational analysis on osteosarcoma patients. Data were taken from osteosarcoma patient's medical record and result of Dynamic Contrast Enhancement in 3 Tesla MRI examination. For ADC we placed the region of interest (ROI) in three places to get ADC value. There were six ROIs from two musculoskeletal radiologist consultants that were averaged. TIC curve were conducted in these ROI and the highest type is pickup. The data was analyzed using SPSS 21 software.

Result: There were 43 samples. Interpretation was conducted by placing three regions of interest (ROI) to determine Apparent Diffusion Coefficient (ADC) value, which was conducted by two musculoskeletal radiologist consultants. Time Intensity Curve (TIC) type was analyzed from DCE-MRI study result. There were $67.4 \%$ male and $32.6 \%$ female samples, where most samples belonged to $11-20$ years group $(53.5 \%)$. The most common osteosarcoma locations were femur $(48.8 \%)$ and tibia $(41.8 \%)$. Osteosarcoma mean ADC value in this study was $\left(1.031 \times 10^{-3} \pm 0.31 \mathrm{~mm}^{2} / \mathrm{s}\right)$, where the highest value was found in chondroblastic subtype $\left(1.470 \times 10^{-3} \pm 0.31 \mathrm{~mm}^{2} / \mathrm{s}\right)$ followed by fibroblastic subtpe $\left(1.003 \times 10^{-3} \pm 0.24 \mathrm{~mm}^{2} / \mathrm{s}\right)$ and osteoblastic subtype $\left(0.984 \times 10^{-3} \pm 0.23 \mathrm{~mm}^{2} / \mathrm{s}\right)$. Osteosarcoma TIC types found in this study were type 3 and type 4 , with predominantly type 3 for all subtypes.

Conclusion: ADC value may improve diagnosis accuracy in osteosarcoma cases. Time Intensity Curve type may also help analysis to diagnose osteosarcoma.
\end{abstract}

Keywords: Osteosarcoma; Apparent Diffusion Coefficient (ADC); Time Intensity Curve (TIC); Osteosarcoma histopathology

\section{Introduction}

Osteosarcoma has a wide range of subtypes based on WHO criteria. Magnetic resonance imaging (MRI) is an important imaging modality for preoperative and post-treatment evaluations of musculoskeletal tumors, especially osteosarcoma. In some cases, conventional MRI might not provide a specific histological diagnosis or determine the extent of tumor necrosis or the presence of viable cells. Thus, advanced MRI techniques for bone tumors are developed and combined with conventional MRI to improve the accuracies of diagnostic, prognostic and treatment response evaluation. Advanced MRI techniques include Dynamic Contrast Enhancement (DCE) perfusion imaging, MR spectroscopy, diffusion weighted imaging (DWI) and apparent diffusion coefficient (ADC) values. ${ }^{1}$

Advanced MRI study using ADC values could differentiate osteosarcoma with its differential diagnosis 
from other bone tumors such as Ewing's sarcoma. ${ }^{2}$ It is difficult to distinguish malignancies, for example osteosarcoma, from osteomyelitis through DWI and ADC values, because they both have restricted diffusion feature on DWI and low ADC value. ${ }^{3}$

Dynamic contrast MR perfusion imaging qualitatively evaluates tumor lesion and tumor-like lesion vascularity by analyzed Time Intensity Curve. Some of malignant tumor shows type 3 and type 4 of TIC. ${ }^{1}$

This study aims to describe the Apparent Diffusion Coefficient (ADC) and Time Intensity Curve (TIC) values in osteosarcoma of histopathological subtypes from Dynamic Contrast Enhancement (DCE)-MRI.

\section{Materials and Methods}

\subsection{Study Design}

This was a retrospective study with observational analysis on osteosarcoma patients' medical records with available MRI Dynamic Contrast Enhancement (DCE) study results at Dr. Soetomo General Hospital, Surabaya, Indonesia from October 2018 - December 2020

\subsection{Ethical clearance}

This study was conducted in accordance with the Declaration of Helsinski. This study was approved by the 100 Medical Research Ethics Committee of Dr. Soetomo General Hospital, Surabaya, Indonesia. All participants included in the samples has given their written informed consent to participate in this study during admission.

\subsection{Data Collection}

Clinical data were taken from the medical records of osteosarcoma patients with available results from advanced MRI study with Dynamic Contrast Enhancement (DCE MRI) and histopathological examinations. Samples were taken from patients who met the inclusion and exclusion criteria that have been determined. MRI study were conducted using Siemens Magnetom Skyra 3 Tesla MRI machine.

MRI study results that were included are coronal, sagittal, and axial sections, T1WI, T2WI, and STIR sequences, DCE-MRI, DWI sequences and ADC. Post-contrast images of conventional FS T1WI on 3 planes were also obtained after dynamic studies.

\subsection{Imaging Protocol}

All study protocols performed on MRI 3 Tesla (Siemens Magnetom Skyra) in axial, coronal, sagital T1-weighted, coronal T2 Fat Saturated and T1 weighted FatSat sequences with contrast on axial, coronal and sagittal sections as well as DWI sequences. DWI and ADC maps were performed on axial plane with $\mathrm{b}$ value $800 \mathrm{~s} / \mathrm{mm}^{2}$, before contrast injection using SS-EPI technique with the following parameters: TR (4430-6640 ms), TE (55-76 ms), , FOV 200-325 mm², matrix size (voxel) 115x128, 5$6 \mathrm{~mm}$ thickness with $1.5 \mathrm{~mm}$ interslice gap, and average 1-2.

\subsection{Imaging Interpretation}

The ROI areas were set to determine the ADC and TIC DCE values. Interpretation of ADC and TIC values was blindly performed by two musculoskeletal radiologist consultants with more than 5 years of experience. To reduce bias, ROI placement was conducted on adequate tissues, therefore it contained only the most enhanced solid components and did not include necrotic components or normal tissue around it (Figure 1). The ROI shape was round or oval with a minimum area of $5 \mathrm{~mm}^{2}$ and a maximum area of $25 \mathrm{~mm}^{2}$. ROI placement on ADC mapping was conducted on axial sections. Three ROIs were selected and ROI placement was intralesional, where DWI and ADC maps were always compared to find the exact viable tumor position, avoiding necrosis and normal tissue areas around the tumor (Figure 1). Three measurements were carried out in ROI area selected by each observer to determine the ADC value. The results were then averaged to obtain the mean $A D C$ value. Mean ADC value from two observers was averaged to be the final mean ADC value. From DCE-MRI study, TIC observations were conducted where TIC type was determined (Figure 1). 


\subsection{Data Analysis}

Data were collected and processed using SPSS 21 software. The mean ADC value from each observer was tested for inter-observer reliability test, where $p<0.05$ and the kappa value $(\kappa)=0.849$ were obtained. These results indicated that the two observers had similar assessment results for ADC value. Apparent Diffusion Coefficient and TIC values from MRI study and histopathological examination results were presented in tables.

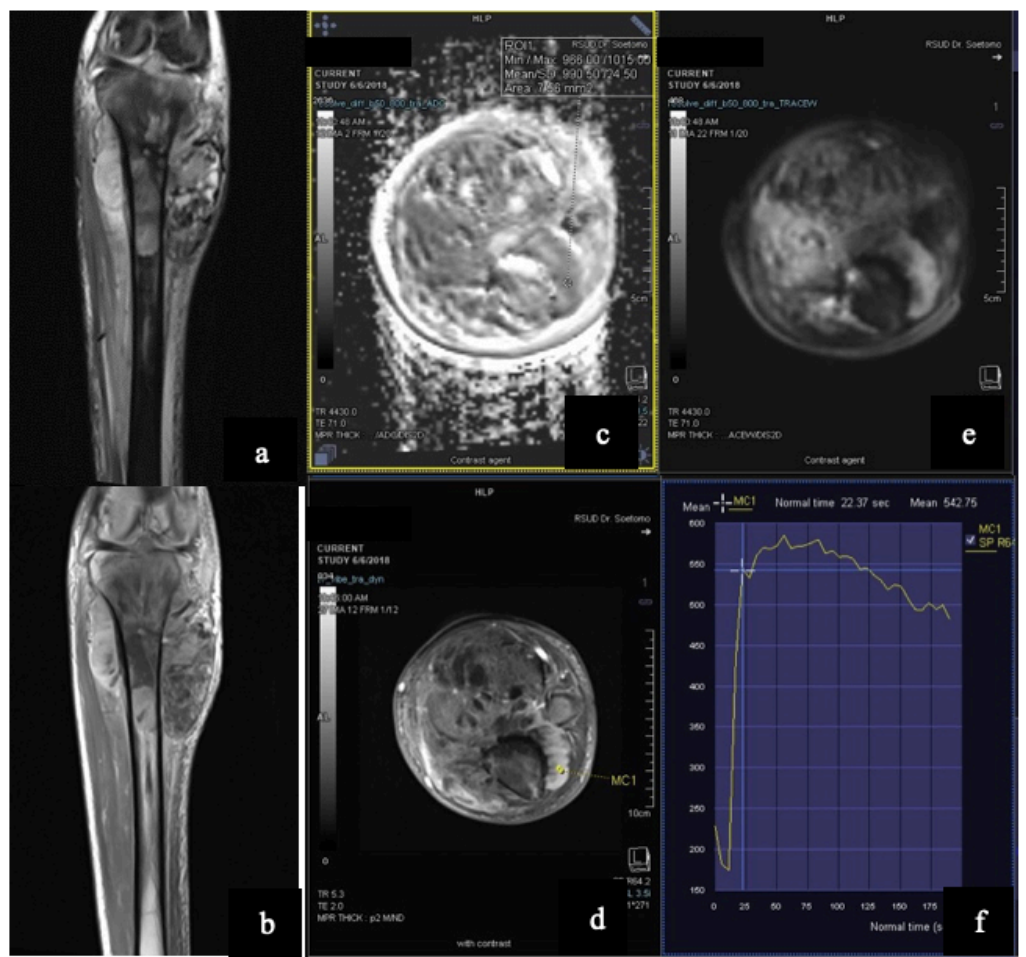

Figure 1. A 19-years-old male with osteosarcoma in proximal right tibia. On MRI examination, solid heterointense mass on Coronal T2 Fatsat (a) and heterogenous contrast enhancement on T1 Fatsat with contrast (b). Restricted diffusion on DWI and ADC (c,e) with ADC value $0.957 \times 10^{-3} \mathrm{~mm}^{2} / \mathrm{s}$. The DCE-MRI showed early contrast enhancement followed by washout showing TIC type $4(\mathrm{~d}, \mathrm{f})$. 


\section{Results}

This study was conducted on 43 samples of osteosarcoma patients. Table 1 outlines the characteristics of study sample. Most of samples were predominantly male with 29 patients (67.4\%), belonged to 11-20 years group with 23 patients (53.5\%), and the most common bone tumor locations were distal femur (48.8\%) and proximal tibia (41.9\%). Most patients were male, in their second decade of life, with femur and tibia as predominant bone tumor location (Table 1). This in accordance with typical characteristics of osteosarcoma. ${ }^{4,8}$

Table 1. Characteristics of Study Samples

\begin{tabular}{clr}
\hline No & Variables & Samples $(\boldsymbol{n}=\mathbf{4 3})$ \\
\hline & Age Group & \\
& 0-10 years & $3(7.0 \%)$ \\
& $11-20$ years & $23(53.5 \%)$ \\
& 21-30 years & $9(20.9 \%)$ \\
& 31-40 years & $4(9.3 \%)$ \\
& 41-50 years & $1(2.3 \%)$ \\
& $>50$ years & $3(7.0 \%)$ \\
\multirow{2}{*}{2} & Gender & \\
& Male & $29(67.4 \%)$ \\
& Female & $14(32.6 \%)$ \\
& Bone Tumor Location & \\
& Distal Femur & $21(48.8 \%)$ \\
& Proximal Tibia & $18(41.9 \%)$ \\
& Fibula & $1(2.3 \%)$ \\
& Humerus & $2(4.7 \%)$ \\
& Pelvic & $1(2.3 \%)$ \\
\hline
\end{tabular}

Osteosarcoma histopathological examination results were classified into several subtypes (Table 2). The most prevalent osteosarcoma subtypes were osteoblastic osteosarcoma in 20 patients (46.5\%), followed by chondroblastic subtype in 8 patients (18.6\%) and giant cell rich subtype in 6 patients (14.0\%). Low grade osteosarcoma is the least sample from all subtype with only one sample.

Table 2. Sample Distribution Based on Osteosarcoma Subtypes

\begin{tabular}{lrr}
\hline Osteosarcoma Subtypes & $\begin{array}{r}\text { Samples } \\
(\mathbf{n}=\mathbf{4 3})\end{array}$ & Percentage (\%) \\
\hline Osteoblastic Osteosarcoma & 20 & 46.5 \\
Chondroblastic Osteosarcoma & 8 & 18.6 \\
Fibroblastic Osteosarcoma & 3 & 7.0 \\
Telangiectasis Osteosarcoma & 3 & 7.0 \\
Small Cell Osteosarcoma & 2 & 4.7 \\
Giant Cell-Rich Osteosarcoma & 6 & 14.0 \\
Low Grade Osteosarcoma & 1 & 2.3 \\
\hline
\end{tabular}


The results of ADC assessments from the two observers were categorized into low and high ADCs. Both observers equally categorized 27 patients to low $\mathrm{ADC}$ and 16 patients to high ADC. The results of these observations were entered into the inter-observer reliability test, where $p<0.05$ and kappa value 0.849 . This result indicates that both observers have the same assessment of the evaluated ADC value.

Table 3. Comparison between Mean ADC Value in This Study and Another Study

\begin{tabular}{lcc}
\hline Osteosarcoma Subtype & $\begin{array}{c}\text { Current Study } \\
\left(\mathrm{x} 10^{-3} \mathrm{~mm}^{2} / \mathrm{s}\right)\end{array}$ & $\begin{array}{c}\text { Zeitoun et al. (2018) } \\
\left(\mathrm{x} 10^{-3} \mathrm{~mm}^{2} / \mathrm{s}\right)\end{array}$ \\
\hline Osteoblastic Osteosarcoma & 0.994 & 1.01 \\
Chondroblastic Osteosarcoma & 1.470 & 1.32 \\
Fibroblastic Osteosarcoma & 1.003 & 1.12 \\
Telangiectasis Osteosarcoma & 0.924 & 1.36 \\
Small Cell Osteosarcoma & 0.735 & 1.11 \\
Giant Cell-Rich Osteosarcoma & 0.817 & - \\
Low Grade Osteosarcoma & 0.746 & - \\
\hline
\end{tabular}

Table 3 compares mean ADC values in current study with another study by Zeitoun et al. Osteosarcoma mean ADC value for all osteosarcoma in our study was $1.035 \pm 0.31 \times 10^{-3} \mathrm{~mm}^{2} / \mathrm{s}$, and the result study by Zeiton et al was $1.16 \pm 0.18 \times 10^{-3} \mathrm{~mm}^{2} / \mathrm{s}^{4}$ The highest mean ADC values our study were found in chondroblastic subtype $\left(1.470 \pm 0.32 \times 10^{-3} \mathrm{~mm}^{2} / \mathrm{s}\right)$, followed by fibroblastic $\left(1.003 \pm 0.25 \times 10^{-3} \mathrm{~mm}^{2} / \mathrm{s}\right)$ and osteoblastic subtype $\left(0.994 \pm 0.24 \times 10^{-3} \mathrm{~mm}^{2} / \mathrm{s}\right)$. The lowest mean ADC value was found in small cell subtype $\left(0.735 \pm 0.7 \times 10^{-3} \mathrm{~mm}^{2} / \mathrm{s}\right)$. Mean ADC values of osteosarcoma subtypes in our study are mostly lower than the results in Zeitoun et al's study with the exception of mean ADC value of chondroblastic type (Table $3)$.

Table 4. TIC Type in Various Osteosarcoma Subtypes

\begin{tabular}{lrr}
\hline \multirow{2}{*}{ Subtype } & \multicolumn{2}{c}{ TIC Type } \\
\cline { 2 - 3 } & \multicolumn{3}{c}{ Type 3 } & Type 4 \\
\hline Osteoblastic Osteosarcoma & $12(60,0 \%)$ & $8(40,0 \%)$ \\
Chondroblastic Osteosarcoma & $7(87,5 \%)$ & $1(12,5 \%)$ \\
Fibroblastic Osteosarcoma & $2(66,7 \%)$ & $1(33,3 \%)$ \\
Telangiectasis Osteosarcoma & $1(33,3 \%)$ & $2(66,7 \%)$ \\
Small Cell Osteosarcoma & $0(0,0 \%)$ & $2(100,0 \%)$ \\
Giant Cell-Rich Osteosarcoma & $3(50,0 \%)$ & $3(50,0 \%)$ \\
Low Grade Osteosarcoma & $1(100,0 \%)$ & $0(0,0 \%)$ \\
Total & $\mathbf{2 6 ( 6 0 , 5 \% )}$ & $\mathbf{1 7}(\mathbf{3 9 , 5 \% )}$ \\
\hline
\end{tabular}

The characteristics of TIC DCE-MRI in various osteosarcoma histopathological subtypes of osteosarcoma are outlined in Table 4. Most subtypes had TIC type 3 with 26 patients $(60.5 \%)$, while TIC type 4 was found in 17 patients (39.5\%). TIC type 3 was mostly found in chondroblastic subtype with 7 patients $(87.5 \%)$ and osteoblastic subtype with 12 patients $(60.0 \%)$, while TIC type 4 was mostly found in telangiectasis subtype with 2 patients (66.7\%). All patients with small cell subtypes had TIC type 4. Meanwhile, all patients with low grade subtypes had TIC type 3. 


\section{Discussion}

Osteosarcoma is a common primary bone malignancy, with the second and third decade as its peak incidence. Osteosarcoma has a wide range of subtypes based on WHO criteria. Magnetic resonance imaging (MRI) is an important imaging modality for preoperative and post-treatment evaluations of osteosarcoma. Advanced MRI techniques for bone tumors are developed and combined with conventional MRI to improve the accuracies of diagnostic, prognostic and treatment response evaluation. ${ }^{1}$ This study aims to describe Apparent Diffusion Coefficient value and Time Intensity Curve in osteosarcoma histopathological subtypes.

From our study sample, most sample belongs to the 11-20 years group or in their second decade of life. The most rapid bone growth is found in this decade. This is in line with osteosarcoma characteristic, which develops in bones with rapid growth. ${ }^{4,5}$

The most common bone tumor locations in this study were distal femur and proximal tibia. The second decade of life in human is puberty phase, where bone grows rapidly. Distal femur and proximal tibia are the bones with the most rapid growth during puberty. 5,6

In this study, the samples were predominantly male. This is because puberty comes earlier in female compared with male, in other words puberty in male comes later compared with female. This might be one of the factors of male predominance in osteosarcoma. ${ }^{6}$

Osteosarcoma is a bone tumor that produces matrices such as osteoid, chondroid or fibrous, with the largest composition being osteoid matrix. This differentiation will determine the histopathological subtype variation. ${ }^{7}$ From our study sample, the most predominant subtype was osteoblastic subtype. This was in accordance with a previous study result. In our study, the mean ADC values in all subtypes were slightly lower compared with a study from Zeitoun et al (Table 5). Osteosarcoma mean ADC value for all osteosarcoma in our study was $1.035 \pm 0.31 \times 10^{-3} \mathrm{~mm}^{2} / \mathrm{s}$, and the result study by Zeiton et al was $1.16 \pm 0.18 \times 10^{-3} \mathrm{~mm}^{2} / \mathrm{s} .{ }^{4}$ Osteoblastic subtype had low mean ADC value. This result was consistent with a study from Zeitoun et al., where they stated that high cellular matrix osteoid resulted restricted diffusion in DWI and low in ADC value. ${ }^{4}$ Another study conducted by Setiawati et al. in 2021 stated that the increase of osteoid matrix between chondroid matrices will increase DWI restriction and decrease ADC value. ${ }^{8}$ The highest mean ADC value was found in chondroblastic subtype. This result was similar to study result.by Setiawati et al. in 2021, where malignant chondrogenic bone tumor mean ADC value was $1.84 \times 10^{-3} \mathrm{~mm}^{2}$ and another study by Yakushiji et al. in 2009 stated that chondroblastic osteosarcoma had higher mean ADC value compared with other osteosarcoma subtypes. This is because tumor cells are more freely dispersed among the chondroid matrices in chondroblastic osteosarcoma, therefore causing a decrease of DWI restriction and an increase of ADC value. ${ }^{9}$

The sample characteristics in our study were predominantly male, in their second decade of life, with distal femur and proximal tibia as predominant bone tumor locations. These characteristics were in line with typical osteosarcoma characteristics. Low mean ADC value and TIC type 3 and 4 represented malignancy characteristics of osteosarcoma.

There are several limitations in this study. First, the number of sample is heterogenous for each osteosarcoma subtypes. Second, ROI placement area to obtain ADC value and TIC type were not confirmed with histopathology specimen sampling area. 
Table 5. Comparison Between Current Study Results with Previous Studies Results

\begin{tabular}{|c|c|c|c|c|c|c|c|}
\hline No. & Author & $\begin{array}{c}\text { Gender } \\
\text { Predominance }\end{array}$ & $\begin{array}{c}\text { Age } \\
\text { Predom } \\
\text { inance }\end{array}$ & $\begin{array}{c}\text { Bone } \\
\text { Tumor } \\
\text { Location } \\
\end{array}$ & $\begin{array}{c}\text { Subtype } \\
\text { Predominance }\end{array}$ & $\begin{array}{l}\text { Mean } \\
\text { ADC }\end{array}$ & TIC Type \\
\hline 1. & $\begin{array}{l}\text { Current } \\
\text { Study }\end{array}$ & $\begin{array}{c}\text { Male } \\
(67.4 \%)\end{array}$ & $\begin{array}{c}11-20 \\
\text { years } \\
(53.5 \%)\end{array}$ & $\begin{array}{l}\text { Right and } \\
\text { left femur } \\
\text { and tibia }\end{array}$ & $\begin{array}{c}\text { Osteoblastic } \\
(46.5 \%)\end{array}$ & $\begin{array}{c}1.035 \pm 0 . \\
31 \times 10^{-3} \\
\mathrm{~mm}^{2} / \mathrm{s}\end{array}$ & Type 3 and 4 \\
\hline 2. & $\begin{array}{c}\text { Jim S. Wu } \\
\text { and Mary G. } \\
\text { Hochman }{ }^{9}\end{array}$ & Male & $\begin{array}{l}10-25 \\
\text { years }\end{array}$ & $\begin{array}{c}\text { Distal } \\
\text { femur and } \\
\text { proximal } \\
\text { tibia }\end{array}$ & - & - & - \\
\hline 3. & $\begin{array}{l}\text { Zeitoun et } \\
\text { al. }(2018)^{4}\end{array}$ & - & - & - & $\begin{array}{l}\text { Osteoblastic } \\
(29.03 \%)\end{array}$ & $\begin{array}{c}1.16 \pm \\
0.18 \mathrm{x} \\
10^{-3} \\
\mathrm{~mm}^{2} / \mathrm{s}\end{array}$ & - \\
\hline 4. & $\begin{array}{c}\text { Costa, } \\
\text { Canella, et } \\
\text { al., }(2011)^{1}\end{array}$ & - & - & - & - & - & $\begin{array}{c}\text { Type } 3 \text { and } 4 \\
\text { in malignant } \\
\text { tumors }\end{array}$ \\
\hline
\end{tabular}

\section{Conclusion}

The mean ADC values in osteosarcoma histopathological subtypes are similar to previous studies. However, there are differences in ADC values in each subtype, especially in osteoblastic type where the value tends to be low, and in chondroblastic type where the value tends to be high. ADC value may improve diagnosis accuracy in osteosarcoma cases. Time Intensity Curve type may also help analysis to diagnose osteosarcoma.

\section{Acknowledgement}

We would like to thank our teachers, my family, and all party who support this study.

\section{Source of Funding}

This study did not receive any specific grant from funding agencies in the public, commercial, or not-for profit sectors

\section{Conflict of Interest}

None

\section{References}

1. Costa, F. M., Canella, C., \& Gasparetto, E. (2011). Advanced magnetic resonance imaging techniques in the evaluation of musculoskeletal tumors. Radiologic Clinics of North America, 49(6), 1325-1358. https://doi.org/10.1016/j.rcl.2011.07.014

2. Pekcevik, Y., Kahya, M., \& Kaya, A. (2013). Diffusion-weighted magnetic resonance imaging in the diagnosis of bone tumors: Preliminary results. Journal of Clinical Imaging Science, 3(1). https://doi.org/10.4103/2156-7514.124094

3. Bhojwani, N., Szpakowski, P., Partovi, S., Maurer, M. H., Grosse, U., von Tengg-Kobligk, H., ZippPartovi, L., Fergus, N., Kosmas, C., Nikolaou, K., \& Robbin, M. R. (2015). Diffusion-weighted imaging in musculoskeletal radiology-clinical applications and future directions. Quantitative Imaging in Medicine and Surgery, 5(5), 740-753. https://doi.org/10.3978/j.issn.2223-4292.2015.07.07

4. Zeitoun, R., Shokry, A. M., Ahmed Khaleel, S., \& Mogahed, S. M. (2018). Osteosarcoma subtypes: Magnetic resonance and quantitative diffusion weighted imaging criteria. Journal of the Egyptian National Cancer Institute, 30(1), 39-44. https://doi.org/10.1016/j.jnci.2018.01.006 
5. Wu, J. S., \& Hochman, M. G. (2012). Bone Tumors A Practical Guide to Imaging. In Bone Tumors; A Practical Guide to Imaging (Vol. 3, Issue 2). Springer

6. Broadhead, M. L., Clark, J. C. M., Myers, D. E., Dass, C. R., \& Choong, P. F. M. (2011). Review Article The Molecular Pathogenesis of Osteosarcoma : A Review. 2011. https://doi.org/10.1155/2011/959248

7. Klein, M. J., \& Siegal, G. P. (2006). Osteosarcoma: Anatomic and histologic variants. American Journal of Clinical Pathology, 125(4), 555-581. https://doi.org/10.1309/UC6KQHLD9LV2KENN

8. Setiawati, R., Suarnata, M. S., Rahardjo, P., Filippo, D. G., \& Guglielmi, G. (2021). Correlation of quantitative diffusion weighted MR imaging between benign, malignant chondrogenic and malignant non-chondrogenic bone tumors with histopathologic type. Heliyon, 7(3), e06402. https://doi.org/10.1016/j.heliyon.2021.e06402

9. Yakushiji, T., Oka, K., Sato, H., Yorimitsu, S., Fujimoto, T., Yamashita, Y., \& Mizuta, H. (2009). Characterization of chondroblastic osteosarcoma: gadolinium-enhanced versus diffusion-weighted MR Imaging. Journal of Magnetic Resonance Imaging, 29(4), 895-900. https://doi.org/10.1002/jmri.21703

10. Gordon, Y., Partovi, S., Müller-Eschner, M., Amarteifio, E., Bäuerle, T., Weber, M.-A., Kauczor, H.U., \& Rengier, F. (2014). Dynamic contrast-enhanced magnetic resonance imaging: fundamentals and application to the evaluation of the peripheral perfusion. Cardiovascular Diagnosis and Therapy, 4(2), 147-14764. https://doi.org/10.3978/j.issn.2223-3652.2014.03.01

11. Jackson, Buckley, and P. (2005). Dynamic Contrast-Enhanced Magnetic Resonance Imaging In Oncology. In Journal of Chemical Information and Modeling (Vol. 53, Issue 9). Springer. 